\title{
Association between preoperative transthoracic echocardiography and clinical outcomes after scheduled hip fracture surgery in geriatric patients
}

\section{Mi-Soon Lee, Sang Hyun Kim, Soonchunhyang University Bucheon Hospital, Soonchunhyang University College of Medicine, Dept of Anaesthesiology \& Pain Medicine, Bucheon, Korea, Republic of}

\section{Background}

Hip fracture has become one of main problem in the elderly patients by progress of the aging society. Transthoracic echocardiography (TTE) is still widely performed for purpose of assessing the risk associated cardiovascular disease in individuals who undergo hip fracture surgery while routine echocardiography was not recommended for the preoperative assessment in individuals undergoing noncardiac surgery by recently published international guidelines. Thus, we evaluated the relevance of the variables of TTE and the outcomes in elderly patients with hip fracture.

\section{Material and Methods}

We conducted single-center, retrospective medical record review including a total of 356 patients. The study population included patients 65 years of age and older underwent TTE within a month before the scheduled hip fracture surgery for the pre-operative assessment between January 2013 and October 2016. Bilateral hip fracture surgery and reoperation due to complication of the previous hip surgery were excluded. Primary outcome was 30-day mortality after surgery, secondary outcomes were postoperative neurologic and cardiovascular complications. Univariable and multivariable logistic regression were performed to identify whether echocardiographic variables are related to postoperative mortality and complications.

\section{Results}

The mean age was 79.7 years $(\mathrm{SD}=6.7)$ and $78(21.9 \%)$ were male. Six patients died within 30 days. There was a significant difference in mean age between the survival group and 30-day mortality group(table1). Delirium was the most common postoperative complication $(46,12.9 \%)$, and cardiovascular disease was the second most common complication (17, 4.8\%).

Among TTE variables only LA volume index was found to be associated with postoperative cardiovascular complication in both the univariable and multivariable logistic regression analysis (Table 2).

\section{Conclusions}

This study shows that patient's age was associated postoperative mortality. More careful screening of the elderly patients for preoperative echocardiography will be necessary.

\begin{tabular}{|c|c|c|c|c|}
\hline Variables & $\begin{array}{l}\text { Total } \\
(\mathrm{N}=356)\end{array}$ & $\begin{array}{l}\text { Survival group } \\
\qquad(\mathrm{N}=350)\end{array}$ & $\begin{array}{l}\text { Death within } 30 \text { day groups } \\
\qquad(\mathrm{N}=6)\end{array}$ & $\mathbf{P}$ \\
\hline Age (year) & $79.7 \pm 6.7$ & $79.6 \pm 6.7$ & $87.5 \pm 3.3$ & 0.003 \\
\hline Gender & & & & 0.616 \\
\hline Male & 78 (21.9\%) & 76 (21.7\%) & $2(33.3 \%)$ & \\
\hline Female & 278 (78.1\%) & 274 (78.3\%) & $4(66.7 \%)$ & \\
\hline Height $(\mathrm{cm})$ & $156.7 \pm 7.9$ & $156.8 \pm 7.8$ & $152.2 \pm 9.2$ & 0.263 \\
\hline Weight (kg) & $54.1 \pm 9.9$ & $54.1 \pm 9.8$ & $52.7 \pm 15.5$ & 0.671 \\
\hline $\mathrm{BSA}\left(\mathrm{m}^{2)}\right.$ & $1.5 \pm 0.2$ & $1.5 \pm 0.2$ & $1.4 \pm 0.2$ & 0.26 \\
\hline \multicolumn{5}{|l|}{ Echo-related factor } \\
\hline LVEF (\%) & $62.8 \pm 7.6$ & $62.7 \pm 7.6$ & $67.3 \pm 7.9$ & 0.161 \\
\hline LV Mass & $135.0 \pm 119.6$ & $135.1 \pm 120.3$ & $129.2 \pm 21.1$ & 0.779 \\
\hline LVMI $\left(\mathrm{g} / \mathrm{m}^{2}\right)$ & $84.5 \pm 23.0$ & $84.5 \pm 23.1$ & $81.3 \pm 5.0$ & 0.945 \\
\hline LAVI $\left(\mathrm{ml} / \mathrm{m}^{2}\right)$ & $24.1 \pm 12.5$ & $24.2 \pm 12.6$ & $23.1 \pm 9.2$ & 0.953 \\
\hline$E(\mathrm{~cm} / \mathrm{sec})$ & $67.9 \pm 18.1$ & $67.9 \pm 18.0$ & $69.6 \pm 22.8$ & 0.99 \\
\hline$A(\mathrm{~cm} / \mathrm{sec})$ & $92.8 \pm 21.3$ & $92.5 \pm 21.0$ & $112.4 \pm 34.4$ & 0.157 \\
\hline $\mathrm{RVP}(\mathrm{mmHg})$ & $32.0 \pm 7.2$ & $32.0 \pm 7.2$ & $33.2 \pm 7.6$ & 0.722 \\
\hline $\mathrm{E}^{\prime}(\mathrm{cm} / \mathrm{sec})$ & $4.8 \pm 1.3$ & $4.8 \pm 1.3$ & $4.0 \pm 1.3$ & 0.141 \\
\hline$A^{\prime}(\mathrm{cm} / \mathrm{sec})$ & $8.6 \pm 1.9$ & $8.6 \pm 1.9$ & $7.8 \pm 2.0$ & 0.419 \\
\hline$E / E^{\prime}$ & $15.0 \pm 6.7$ & $15.0 \pm 6.7$ & $18.5 \pm 6.5$ & 0.176 \\
\hline \multicolumn{5}{|l|}{ Comorbidity } \\
\hline Hypertension & 224 (62.9\%) & 221 (63.1\%) & $3(50.0 \%)$ & 0.674 \\
\hline Diabetes mellitus & $108(30.3 \%)$ & 106 (30.3\%) & $2(33.3 \%)$ & 1 \\
\hline Coronary arterial disease & $37(10.4 \%)$ & $36(10.3 \%)$ & $1(16.7 \%)$ & 0.485 \\
\hline Heart failure & $22(6.2 \%)$ & $20(5.7 \%)$ & $2(33.3 \%)$ & 0.047 \\
\hline Valvular disease & $9(2.5 \%)$ & $9(2.6 \%)$ & $0(0.0 \%)$ & 1 \\
\hline Arrhythmia & $20(5.6 \%)$ & $19(5.4 \%)$ & $1(16.7 \%)$ & 0.295 \\
\hline Stroke & 65 (18.3\%) & $63(18.0 \%)$ & $2(33.3 \%)$ & 0.302 \\
\hline Neurologic disease & $83(23.3 \%)$ & $81(23.1 \%)$ & $2(33.3 \%)$ & 0.627 \\
\hline Kidney disease & $35(9.8 \%)$ & $33(9.4 \%)$ & $2(33.3 \%)$ & 0.11 \\
\hline Pulmonary disease & $57(16.0 \%)$ & 56 (16.0\%) & $1(16.7 \%)$ & 1 \\
\hline
\end{tabular}

A. mitral peck velocity of late filling; $B S A$, body surface area; $E$, mitral peak velocity of early filling; $E^{\prime}$, early diastolic mitral annular velocity of the septal mitral annulus; $E / E^{\prime}$, the ration of mitral peak velocity of early filling(E) to early diastolic mitral annular velocity of the septal mitral annulus(E'); LAVI, left atrial volume index; LVEF, left ventricular ejection fraction; LV Mass, left ventricular mass; LVMI, left ventricular mass index; LV

Table 1. Demographic characteristics, echocardiologic findings and patient's cormobities

\begin{tabular}{|c|c|c|c|c|c|c|c|c|c|}
\hline & \multicolumn{3}{|c|}{ Death within 30 days } & \multicolumn{3}{|c|}{ Delirium } & \multicolumn{3}{|c|}{ Cardiovascular complication } \\
\hline & OR (\%) & $95 \%$ of $\mathrm{Cl}$ of $\mathrm{OR}$ & $\mathrm{P}$ & OR (\%) & $95 \%$ of $\mathrm{Cl}$ of $\mathrm{OR}$ & $\mathrm{P}$ & Odd ratio & $95 \%$ of $\mathrm{Cl}$ of $\mathrm{OR}$ & $\mathrm{P}$ \\
\hline \multicolumn{10}{|c|}{ Univariable regression analysis } \\
\hline \multicolumn{10}{|c|}{ Transthoracic echocardiographic variables } \\
\hline $\operatorname{LVEF}(\%)$ & 1.1 & $0.98-1.25$ & 0.132 & 0.96 & $0.91-1.02$ & 0.21 & 1 & $0.96-1.04$ & 0.871 \\
\hline LVMI (g/m2) & 0.99 & $0.95-1.04$ & 0.809 & 1.01 & $0.99-1.03$ & 0.409 & 1.01 & $0.99-1.02$ & 0.455 \\
\hline LAVI (ml/m2) & 0.99 & $0.88-1.06$ & 0.885 & 1.05 & $1.02-1.09$ & 0.006 & 0.99 & $0.95-1.03$ & 0.764 \\
\hline $\mathrm{E}(\mathrm{cm} / \mathrm{sec})$ & 1.01 & $0.95-1.05$ & 0.831 & 1.01 & $0.98-1.04$ & 0.471 & 0.99 & $0.97-1.01$ & 0.575 \\
\hline $\mathrm{A}(\mathrm{cm} / \mathrm{sec})$ & 1.03 & $1-1.06$ & 0.044 & 0.99 & $0.96-1.02$ & 0.493 & 1 & $0.98-1.01$ & 0.874 \\
\hline RVP mmHg) & 1.02 & $0.88-1.12$ & 0.748 & 1 & $0.92-1.07$ & 0.995 & 1 & $0.95-1.05$ & 0.85 \\
\hline $\mathrm{E}^{\prime}(\mathrm{cm} / \mathrm{sec})$ & 0.52 & $0.2-1.13$ & 0.131 & 1.15 & $0.64-1.65$ & 0.511 & 0.04 & NA-10236423.95 & 0.99 \\
\hline$E / E^{\prime}$ & 1.04 & $0.94-1.11$ & 0.258 & 0.97 & $0.87-1.05$ & 0.585 & 0.99 & $0.94-1.04$ & 0.748 \\
\hline Age & 1.21 & $1.06-0.4$ & 0.007 & 1.01 & $0.97-1.06$ & 0.572 & 1.06 & $0.99-1.44$ & 0.122 \\
\hline \multicolumn{10}{|c|}{ Multivariable regression analysis } \\
\hline Age & 1.19 & $1.03-1.36$ & 0.016 & - & - & - & - & - & - \\
\hline LAVI (ml/m2) & - & - & - & - & - & - & 1.21 & $1.03-1.42$ & 0.02 \\
\hline
\end{tabular}

$E^{\prime}$, early diastolic mitral annular velocity of the septal mitral annulus; $E / E^{\prime}$, the ration of mitral peak velocity of early filling(E) to early diastolic mitral annular velocity of the septal mitral annulus( $\left.E^{\prime}\right)$; LAVI, left atrial volume index; LVEF, left ventricular ejection fraction; LV Mass, left ventricular mass; LVMI, left ventricular mass index

Table 2. Results of the univariable and multivariable regression analysis of between the transthoracic echocardiographic variables and the 30-days mortality and the postoperative neurologic and cardiovascular complications 